\title{
Exploration of Propensity for Using Acrolect in Code-switching among the Youths of Bangladesh: A Psychological Study of Bilingualism
}

\author{
Md. Nahid Hasan Neloy*, Dipak Kumar Sarkar² \\ ${ }^{\prime}$ Department of English, Hajee Mohammad Danesh Science \& Technology University, Bangladesh \\ ${ }^{2}$ Assistant Professor, Department of English, Hajee Mohammad Danesh Science \& Technology University, Bangladesh
}

Corresponding Author: Md. Nahid Hasan Neloy, E-mail: neloy.eng.hstu@gmail.com

\section{ARTICLE INFO}

Article history

Received: September 14, 2019

Accepted: November 18, 2019

Published: December 31, 2019

Volume: 10 Issue: 6

Advance access: December, 2019

Conflicts of interest: None

Funding: None

\section{Key words:}

Code-switching,

Acrolect,

Youth,

Propensity,

Sociolinguistics,

Psychology,

Bilingualism

\begin{abstract}
This paper aims to uncover the phenomenon of Code-switching and the motivation behind the propensity of using acrolect in code-switching among the youths of Bangladesh. In comparison to the past, switching code has become an ecumenical term of Bilingualism in this world. Countries like Bangladesh where English has been taught or used as a foreign language, people often switch the code from Bengali to English or English to Bengali. The tendency of switching code is seen mostly in the youths and young professionals in Bangladesh. Moreover, it is seen the inclination of preferring acrolect while switching the code. The paper analyzes how the youths and young professionals can safely adapt code-switching and make a positive impression on it. The study consists of both qualitative and quantitative approaches. To analyze the nature and factors of code-switching, the paper takes help from different sources and some surveys and questionnaires are completed for empirical data collection. To answer the questions, it is compared to the answers of some students and teachers from Hajee Mohammad Danesh Science \& Technology University, Dinajpur and other reputed universities in Bangladesh as well. The paper will trace the role of switching code and its consequences on Bangladeshi youngsters. And the findings can be used in Sociolinguistic ground to expand the nature and scope of codeswitching. The result shows an unleashing effect of code-switching and the positive and negative attitude towards code-switching. The results answer how acrolect dominates over a speaker while switching the code and it also answers regarding the correlation between proximity to code-switching and psychology of the youths.
\end{abstract}

\section{INTRODUCTION}

It would not be surprising if it is said that a hundred percent of the youths are used to switching the code in their daily conversation. Perhaps, Language plays an important role to express thoughts, ideas, or emotions. In the 21 st century, Code Switching has become a very common phenomenon in Bangladesh for globalization and technological propagation. Besides, People frequently switch the code from English to Bengali or vice-versa and Bengali to Hindi or even vice-versa etc. Even the Code-Switching can be called as the juxtaposition between two languages in conversation. According to R. A. Hudson, Code Switching is preferred to say 'language -switching'. Mostly, Code Switching depends on the particular situation that is why it is also called 'situational code-switching'. In other words, Code Switching can be defined as an alternation between two or more languages in a conversation that happens in the speech of Bilinguals. With the advancement of modernism and post-modernism, switching code has become a trend among the youths. In the context of Bangladesh, Code-switching has become a fashion or a habit like taking a tea without any reason. Family background, social class, religious norms, and some other inevitable circumstances make code-switching very frequent. Though Bangladesh is not a fully Bilingual country, its young people and others have adopted it very compactly. Sometimes, Code-switching happens for a better understanding and so it is co-related with the psychology of the speaker. It is also seen that youngsters switch code unnecessarily and unknowingly. Moreover, in Universities or Colleges, young students are more contextualized in switching the code. TV, Radio, and other social media are also responsible for this code-switching where entertainment is latent in the Hindi movies or other popular TV shows. Increasing English medium schools, online schools emphasise more on the English language proficiency which leads a young to switch the code. For all these reasons, it is seen a drastic change in the style of talking. These entire hypotheses can be proved as wrong. Hence, this paper is going to discuss and find out the reason behind it. Eventually, there is a close relation between code-switching and the psychology of the youngsters. They, usually think that switching code may be a 'smartness' or this is a 'trend'. Different psychological aspects will have been 
studied in this paper. This paper approaches mostly about the function and application of code-switching in different places and in the later part it will discuss about some psychological discourse. By performing some empirical data analysis, the study will find the real motivation behind code-switching. The aim and goals of the paper are to trace out the phenomenon of why code-switching, regardless, and necessary where acrolect works as a catalyst in code-switching.

\section{Objective}

The objective of the study is to find out the inherent phenomenon of using acrolect in code-switching based on the sociolinguistic ground of study. It also intends to examine the data to analysis the code-switching tendency among the emerging youths and the repercussion of code-switching in individual sectors. The study will determine what things could be done to make the code-switching feasible in different sectors. The paper attempts to clarify the whole idea of code-switching to make people understand when and how the code-switching can be done or be avoided. Hence, the allied objective can be found for a better understanding of the nature and scope of code-switching in the Bangladeshi context.

\section{Methodology}

To do the study, both qualitative and quantitative methods have been chosen. To illustrate the behaviour and patterns of code-switching among the youths, the qualitative method has been adopted. Interview and questionnaire have been done among 20 students and 5 teachers from Hajee Mohammad Danesh Science and Technology University, Dinajpur and other reputed universities in Bangladesh. All the students and teachers are from different disciplines. It also includes more than 10 students from the HSC level belonging to different regions of Bangladesh. Moreover, different types of open-ended questions are asked to find out the real nature of code-switching. This study also includes a quantitative approach. From quantitative data collection, the collected data has been used to calculate the percentage of code-switching and the answers give a complete idea about code-switching propensity among the youngsters. For analytical information, a comprehensive review of the literature has been done. However, this study also takes help from different books, articles, online articles, journals, etc. So the study configuration goes with both primary and secondary sources.

\section{Rationale}

The topic is chosen because of ascertaining a lucid notion about code-switching tendency, particularly among the Bangladeshi emerging youths. Previously, in this topic, a lot of investigations have already been accomplished but this paper aims only on the Bangladeshi context. The increasing zeal for code-switching in Bangladesh makes the topic worth studying. The amalgamation of the theoretical perspective and qualitative approach has timbered the study credible and realistic. The analysis of the study will help both the teachers and students to find out how frequently switching the code is safe and acceptable regarding educational partition.

\section{Problem Statement}

The whole paper deals with some particular problems. Firstly, it questions why youths usually switch the code as all of them have a native language and most importantly why they prefer to use acrolect in code-switching. Secondly, whether code-switching is an issue of social acceptance or not. It also comes up with the question of switching code might make cultural diversity or not. Thirdly, it also investigates how code-switching is a part of comfort zone or a matter of problem. Fourthly, it gives light on the adaptation problems of code-switching. Finally, the code-switching and code-mixing confusion will be ventilated in this paper.

\section{LITERATURE REVIEW}

\section{Code-switching}

According to R. A. Hudson, "Code-Switching, which is the inevitable consequence of bilingualism (or, more generally, multilingualism). Anyone who speaks more than one language chooses between them according to circumstances. The first consideration, 'of course', is which language will be comprehensible to the person addressed; speakers choose a language which the other person can understand." (Hudson 2007:51).

Chui, Liu, and Mak (2014) propose, "Code- switching is the oral or written switching between distinct varieties across sentential, clausal, phrasal, or lexical boundaries. The determination of code choice in a conversation depends on the socio-cultural background of participants." (Chui, Liu, \& Mak 2014).

\section{Acrolect}

In sociolinguistics, acrolect is a creole variety that tends to command respect because its grammatical structures do not deviate significantly from those of the standard variety of the language. The term acrolect was introduced in the 1960s by William A. Stewart and later popularized by linguist Derek Bickerton in Dynamics of a Creole System (Cambridge Univ. Press, 1975). Taking decreolisation first, this is what happens when a Creole is spoken in a country where other people speak the Creole's lexical source-language (for example, English). Since the latter has so much more prestige than the Creole, Creole speakers tend to shift towards it, producing a range of intermediate varieties. Sociolinguists call the Creole the basilect and the prestige language the acrolect. (Hudson 2007: 65).

\section{Conversational Code-switching}

According to Gumperz (1982), "Conversational code-switching can be defined as the juxtaposition within the same speech exchange of passage of speech belonging to two different grammatical system or subsystems." (Gumperz 59). 


\section{Types of Code-switching}

Inter-sentential switching occurs outside the sentence or the clause level. It is sometimes called "extra-sentential" switching. In inter-sentential code switching, the language switch is done at sentence boundaries - words or phrases at the beginning or end of a sentence. This type is seen most often in fluent bilingual speakers.

Intra-sentential switching occurs within a sentence or a clause. In intra-sentential code switching, the shift is done in the middle of a sentence, with no interruptions, hesitations, or pauses to indicate a shift. The speaker is usually unaware of the shift. Different types of switches occur within the clause level and within the word level.

Tag-switching is the switching of either a tag phrase or a word, or both, from one language to another. This is the switching of either a single word or a tag phrase (or both) from one language to another. This type is common in intra-sentential switches. It involves the insertion of a tag from one language into an utterance in another language

Intra-word switching occurs within a word itself, such as at a morpheme boundary.

Most code-switching studies primarily focus on intra-sentential switching, as it creates many hybrid grammar structures that require explanation. The other types involve utterances that simply follow the grammar of one language or the other. Intra-sentential switching can be alternational or insertional. In alternational code-switching, a new grammar emerges that is a combination of the grammars of the two languages involved. Insertional code-switching involves "the insertion of elements from one language into the morpho-syntactic frame of the other" (Wei 2000).

\section{Reason behind Code-switching}

Holmes (2001) talks about the reason behind code-switching and he says, "People sometimes switch code within a domain or social situation. When there is some obvious change in the situation, such as arrival of a new person, it is easy to explain the switch." She also tells that people often switch the code when anyone meets with a new person. Therefore, Holmes argues that, Code-switching is related to a particular participant or an addressee.Holmes also claims that people usually switch codes subconsciously that is they are not conscious of the fact that they are mixing or, switching to another language. If they are conscious of this behavioral fact, they sometimes apologize for mixing which indicates disapproval of mixing another language. It sometimes, possible that due to "ethnic self-consciousness" manipulation of another language indicates this disapproval of switching or mixing code. (Holmes 35,45).

According to Wardhaugh (2010), "A diglossic situation exists in a society when it has two distinct codes which show clear functional separation: that is, one code is employed in one set of circumstances and the other in an entirely different set. Wardhaugh says, "Functioning in a diglossic situation requires a person to use two codes" as "functioning in a single variety of language would appear to be an extremely rare phenomenon." In diglossic communities, the situation dominates the selection of variety, but the choice is much more rigidly determined by the particular activity that is involved in and the relation between the participants. Diglossic situation reinforces difference that is socially approved, for example, conventional or official function. But code-switching tends to reduce such a diglossic situation. In diglossic circumstances, people are conscious that they are shifting from high to low or low to high; whereas during code-switching, people are not aware that they have switched, or be able to report, following a conversation, which code they are using for a particular topic." (Wardhaugh 90,102).

According to Vogt(1954), "Code-switching is not only natural, but common. He suggested that all language-if not all language users-experience language contact, and the contact phenomena, including language alternation, are an important element of language change." (qtd. in Nilep 22).

P. Auer (1998) identified eight conversational loci in which code-switching are frequent: a) reported speech, b) change of participant constellation (address selection and the use of code-switching to include/exclude/marginalize participants or bystanders), c) Parentheses or side comments, d) reiterations (quasi-translations into the other language for putting emphasis on demands, requests, for clarification, attracting attention and the regulation of turn-taking), e)change of activity type (also referred to as mode shift or role shift), f)topic shift, g) puns, language play and shift of key, h) topicalization and topic/comment structure. Based on previous research, all these factors influence bilinguals to switch codes. But it does not necessarily mean that every one of these factors will be active during code-switching. Rather different factors will be influencing bilinguals at different times. Now, it should be mentioned that code-switching can be of three types. Each type occurs in a diverse situation which sometimes even helps us to identify whether a bilingual is "balanced" or "non-balanced". Poplack (2000) stated, "balanced bilingual" will display a noticeable preference for what she calls the "more complex or "intimate" type of code-switching. non-balanced bilinguals (e.g., speakers who have acquired the second language later in life) supposedly prefer what Poplack terms "emblematic" code-switching, i.e. tag switches and single-noun switches (which are often hard to distinguish from lexical borrowings) .It can occur within sentences (intra-sentential); between sentences (inter-sentential) or on extra-sentential level that indicates an occurrence in which a bilingual may attach a tag from one language code into a statement in another code, alternatively known as tag switching. (ctd. In Inuwa,et al. 2014:44).

\section{Factors Influencing Code-Switching}

Grosjean in his book, Life with Two Language listed some factors influencing code choice. It is given below- (Grosjean 1982:136).

\begin{tabular}{ll}
\hline Participants & Situation \\
\hline Language proficiency & Location/setting \\
Language preference & Presence of monolinguals \\
Socioeconomic status & Degree of formality \\
Age & Degree of Intimacy \\
Sex & Content of discourse \\
Occupation & \\
\hline
\end{tabular}




\section{Language and Mind}

Noam Chomsky in his book Language and Mind says, "The person who has acquired knowledge of a language has internalized a system of rules that relate sound and meaning in a particular way. The linguist constructing a grammar of a language is in effect proposing a hypothesis concerning this internalized system. The linguist's hypothesis, if presented with sufficient explicitness and precision, will have certain empirical consequences with regard to the form of utterances and their interpretations by the native speaker. Evidently, knowledge of language - the internalized system of rules - is only one of the many factors that determine how an utterance will be used or understood in a particular situation. The linguist who is trying to determine what constitutes knowledge of a language - to construct a correct grammar - is studying one fundamental factor that is involved in performance, but not the only one. This idealization must be kept in mind when one is considering the problem of confirmation of grammars on the basis of empirical evidence. There is no reason why one should not also study the interaction of several factors involved in complex mental acts and underlying actual performance, but such a study is not likely to proceed very far unless the separate factors are themselves fairly well understood." (Chomsky 2006:23).

\section{Code-switching in Bilingual and Multilingual Situation}

Wardhaugh (2010) illustrates some of the contexts of bilingual and multilingual communities around the world. A community like Montreal and Quebec where two languages are coexisting in everyday lifestyle is a bilingual community. Generally, one code is used to maintain the communication within the community and another is outside the community to express identity, solidarity, status, etc. A bilingual speaker may insist on using a particular group if it is not within the community, for example, a French Canadian may insist on using French to an official of the federal government outside Quebec. In a multilingual community or country, the ability to shift from one language to another is accepted as quite normal. A country like Singapore with four official languages offers a wide choice among languages, with an actual choice determined by the different context of the country for example, at home, at the workplace, at school, in trade, or a distinct ethnic community. (Wardhaugh 98-101).

\section{Code-switching in Bangladesh}

Banu and Sussex (2001) talk about the linguistic background of Bangladesh as they show the long tradition of code-mixing and show the code-switching in the naming of shops and business centers. They also shed light on the fact that Bangladesh was long under colonial regime, when English was considered as a high variety of language. Then again, after independence from British Bangladesh came under the rule of Pakistan (then West Pakistan). Even at that period the Pakistanis declared Urdu as a state language, not Bengali. However, after the blood shedding language movement of 1952, Bengali also get the status of state language of Pakistan. Even after that, the period between language movement and liberation war in 1971, both Urdu and English had great influence in Bangladesh. It is only after the independence of Bangladesh, all of a sudden people started to look at both Urdu and English with contempt in their eyes. Names of shops, roads, and schools were changed overnight. Some places were vandalized as well. However, they also mention that English is once again starting to re-enter the lives of former colonial countries. (Banu and Sussex 52-53).

The history of English in the Bengali language has stepped in during the colonization in Bangladesh which was then a portion of East India Company. Some of the common words like office, hospital, chair, table, and so many different words of English have become the Bengali language that it is not even noticed while using. Now, both the government and private sectors require good command over the English language. In educational sectors, there are also English medium and English version categories, and English is taught as a compulsory subject in every level of education from primary to tertiary level.

Nargis Chowdhury, in her paper talks about class room code-switching in Bangladesh. According to her, Bangladesh is a monolingual country and the medium of study is Bengali in primary and secondary level of education mostly. But English has a growing demand in Bangladesh and being a part of the global community the necessity and importance to learn English is felt more and more day by day. Hence, in the English medium schools, and English version schools are growing up. But sometimes, in the university classroom, it is found that mother tongue interference as a common fact. This phenomenon creates scopes for classroom code-switching. (Chowdhury 2013).

\section{CODE-SWITCHING AND RELATED PHENOMENON}

\section{Language and Code-switching}

As code-switching is related to language, there is a huge impact on language change over code-switching. Being a bilingual speaker, people usually switch the code very frequently. A person who knows more than one language, habitually, switches the code. For example- an English lecturer of a university who frequently switches the code in the classroom or other circumstances. Sometimes, he switches the code to make his students familiar with a new theory or a literary device. For the instance of teaching a literary term called 'Paradox' at first, the teacher talks about the literary devices in English (like the definition) then for better understanding with some sophisticated example he tends to use the Bengali language. That is the way by which code-switching takes place in daily life conversation.

Code-switching happens not only between Bengali and English. Furthermore, code-switching takes place between Arabic and Bengali also. It is a very common scenario in Bangladesh that in the Mosque the Muslim "Imam" ( Who leads in the Mosque) who switches the code over and over. While giving lectures in the Mosque, he usually tells an "ayat"(Holy verse of Quran) in Arabic language and then he explains it in the Bengali language for a better understanding. Here comes the term 'diglossia' which is "a relatively stable language situation in which, in addition to primary dialects of the language, there is a very divergent, highly codified, superposed variety, the vehicle of a large and respected body of written 
literature, either of an earlier period or in another speech community, which is largely learned by formal education and is used for most written and formal spoken purposes but is not used by any sector of the community for ordinary conversation." (Hudson 2007:49-50) Arabic is like having a diglossic situation that is the language that is highly codified in the Bangladeshi context. That is why; it is needed to shift the code from Arabic to Bengali for a better understanding of the entire phenomenon regarding Muslim religion.

Like English and Arabic, Code Switching happens between Hindi and Bengali language. The young boys and girls who are very popular with Bollywood movies and Indian drama serials use to switch the code very often. It is seen that the young boys and girls are used to use Hindi phrases or dialogues of Hindi movies in informal circumstances. Hence, if a speaker craves to switch the code; has to know more than one language. That means $s /$ he has to be a bilingual or multilingual speaker.

\section{Youths and Code-switching}

The study mainly aims to shed light on the code-switching of the emerging youths of Bangladesh. Why and how they switch the code that is the question. To answer this question, Suraiya Alam says in her research paper that there have several reasons behind the code-switching of the young's. Firstly, she says that young people switch the code because of keeping the spontaneity. Besides, to impress the opposite sex, mostly, the youngsters are tending to switch the code very often. The data analysis says that about $21 \%$ of code-switching takes place to impress the opposite sex. She also suggested that code-switching happens also for "euphemism'. In Bangladesh, it is very frequent that people used to shift the code when some odd words come to the situation. (Alam 2006:57-59)

The young also tends to switch the code for professional purposes. If anyone switches the code in Bangladesh, it is thought that $\mathrm{s} / \mathrm{he}$ is smarter than any other young. It is a tool of showing off also. This is a very common scene which is seen in the corporate or other job sectors. Lack of equivalent diction, sometimes, is seen among the young who are prone to switching the code. Hence, it is said that, for being a part of the so-called smart society, the young tend to switch the code.

\section{Code-switching and Media}

\section{English code-switching in Bangladeshi commercial cinema}

Sultana (2010) in her paper illustrates that code-switching in cinema is done in dialogue with a reason to convey some meaning, and she finds out some of the reasons and types of code-switching in Bangladeshi commercial films from an academic point of view. According to her study, there are some obvious reasons for code-switching in every cinema that she has observed. These are: habituation, to show high status, to raise humour/comedy, to maintain official norms/ situational norms, to show being educated, to show authority, to show respect according to religion. From her analysis, it is found that "in films, code-switching occurs as a result of mere habituation of the speakers." Primarily, English is used in switching the codes from Bengali to show the characters are educated. It is observed that most of the characters are comfortable in mixing Bengali-English during their conversation with each other, so it does not seem artificial or imposed. Usually, filler words, tag words, common English nouns or words are used in mixing the code." Again "Bengali to English code-switching occurs when someone wants to emphasize on a particular dialogue or expression. The English language is used to accomplish the desired meaning in the conversation. This code-switching is done in two ways: the characters pronounce the same dialogue two times in English to put more emphasis, and the other is once the dialogue is said in Bengali and then again the same dialogue is said in English.” (Sultana 40-43).

\section{Code-switching in university classroom}

In the university classroom, code-switching is seen very frequently. Bilingual speakers shift the codes for various reasons during the conversations. In university classrooms, code-switching comes into use in case of both the teachers' and the students' discourse. Sometimes the teacher shifts code in a bilingual classroom based on the situation, revising and repeating to clarify a point. It is encountered that to handle bilingual instructional materials and methodology, teachers will ideally have to be balanced bilinguals who can switch with ease between languages. This is why researchers believe that code-switching is not essentially a barrier or a deficiency in learning as well as teaching. The use of both languages creates a diglossic environment and ultimately produces code-switching. According to Banu and Sussex, as the use of English is increasing day by day in different forms, there is significant evidence of the use of English along with Bengali as code-mixing and code-switching. The use of more than one language in the university classroom creates code-switching.

Two kinds of code-switching varieties are seen in the university classroom of Bangladesh. These are

1. Bengali to English (B2E) code-switching: When Bengali is used as the medium of instruction, using English alongside Bengali in the classroom will result in code-switching; this is called Bengali to English (B2E) code-switching.

2. English to Bengali (E2B) code-switching: If the English language is used as the medium of instruction, using the Bengali language in the class, instead of the English language, will be considered as English to Bengali (E2B) code-switching. (Banu and Sussex 2001).

\section{Code-switching in social media}

Young generation are very familiar with social media and so they are habituated to utilizing the social media in that ways that they cannot even stay a single day without social media. And in these social media it is very frequently seen to switch the code by its users. 'Facebook' has gained tremendous popularity as a social media text. A large number of people use Facebook to inter- 
act, get information, share different things, and give personal comments. Although, all the instructions and applications are in English, Bangladeshi young people tend to use both Bengali and English to correspond on Facebook. It has been figured out that almost $100 \%$ of people who communicate on Facebook are mostly friends and colleagues and they find "it easy to communicate that way" and it happens mainly "for suitable words" and "to put emphasis" and it is "spontaneous". It is found out the fact that "most of the Bangladeshi users switch code because it has become spontaneous" and "switching codes help them to convey their message in a better way, especially when they cannot find a word in one language, they switch to others to keep the flow of the conversation." And the use of language depends on the following two factors: topic and how others reply Koziol (2000) also manifest some functions of code-switching such as personalization, reiteration, designation, substitution, emphasis, clarification, objectification, aggravating messages, interjections, parenthesis, quotation, and topic shift. According to her view, these are due to the speaker's effort to personalize messages for their listeners. Like Facebook in other social media, code-switching takes place very frequently. (Kaziol 2000).

\section{Code-switching in FM radio}

According to S. Ahmed and F. Z. Tinny (2011) combination of Bengali and English were applied to practice casually among friends in the university campus or other places where young people gather mostly but nowadays it is being used formally in almost every gathering and the media, "especially the FM radio is greatly impacted by it. It is said that these FM channels are promoting this new style of speaking and spreading widely among the young generation". And as the new and young generations have developed a choice of following a new style or fashion or way of speaking, eventually, their subculture is taking over the natural way of speaking Bengali. From her research, it is evident that $84.5 \%$ of students are familiar with this new style and $54 \%$ are interested in using the new style and their $20 \%$ and $16 \%$ sources are from the radio and TV channels. (Ahmed and Tinny 3 ).

\section{Code-switching in Group-chat}

The youths are very enthusiastic and it can be seen that they often go for hangout, party and group-chat. The situation can be defined as informal. And the young usually used to switch the code in their group-chat. They feel very comfortable to switch the code in this type of circumstances. Some examples of code-switching in group chat are given below-

1) This is final $\mathrm{j}$ kal $\mathrm{k}$ amra ei kaj ti korbo (that we will do this work tomorrow).

2) We will meet next Friday, thik ache? (shall we?)

3) Taratari meeting ta ashes kor (Finish the meeting in short). I am getting late.

4) Amakeei Burger ta khete de (Let me eat this Burger). This is my favorite.

5) Shobai kal k ashba (Everybody is invited to come tomorrow). Am I clear?

From the upper examples, it can be seen that the young, sometimes, switch the code from Bengali to English or
English to Bengali. And it has made a habit of talking and this habit is formed according to various circumstances. The students who have come from an English based school switch the code more frequently.

\section{Arena of Code-switching}

From a hypothesis, it can be assumed that there are some possible arenas where the code-switching can be seen frequently. These are listed below:

- University classroom

- Radio and Television

- Official party

- Mosque

- Speech

- Writing SMS

- Group chat

- Songs

\section{Patterns of Code-switching}

\section{Bengali and English}

(a) Stop writing. Somoy shes (the time is over).

(b) You have to promise tumi amake help korbe (you will help me).

(c) Shakespeare bolechen tar Hamlet natoke (has said in his drama Hamlet) "Frailty thy name is women."

(d) I am very sorry. Amar ata kora uchit hoy ni (I should not have done it).

(e) If you look at the nature you will get a pleasure. Sotti ata osadharon(Really it is amazing).

(f) It's ok. Amar kono tara nei (I have no urgency).

\section{Hindi and Bengali}

(a) Kemon acho? (How are you?) Keya chal raha hain (How is going everything?)

(b) Alga koro go khopar badhon (Please, loosen the looping frot of yours), dil wahi mera fas geyi (my mind is stucked there).

\section{Arabic and Bengali}

(a) Ami kaj ti korbo (I will do the work) Inshallah (If Allah permits me).

(b) Fiamanillah (By the grace of Allah) tumi valo korbe (you will do better).

\section{DATA COLLECTION METHODS AND DATA ANALYSIS}

\section{Participants of the Study}

Some empirical data collection is been conducted here. A questionnaire section is set up and interviews have been taken for this paper. Approximately, 20 students are asked several questions and interview has been done with 5 teachers from Hajee Mohammad Danesh Science and Technology University, Dinajpur and other reputed universities as well 
in Bangladesh. Besides, some open ended questions are also asked to know about theirexperience, thinking, and ideology.

\section{Questionnaire Method}

To illustrate the pattern of code-switching and to find out the inherent elements of code-switching a questionnaire section is set up. Different types of questions are asked in this section. They are given below and the result is shown through pie chart.

Question no.1- Do you switch the code?

Data analysis: When this question is asked among 20 students, they answered it after understanding the term code-switching. Here, it can be seen that $75 \%$ of students have answered "yes", and $15 \%$ of them answered "No". Whereas, others don't have any particular answer because either they don't catch the term code-switching or they were confused (See Figure 1).

Question no. 2- Do you think that Code-switching happens frequently?

Data analysis: In this question, most of them (70\%) have answered positively saying that it happens frequently. And a portion of $25 \%$ of students have answered

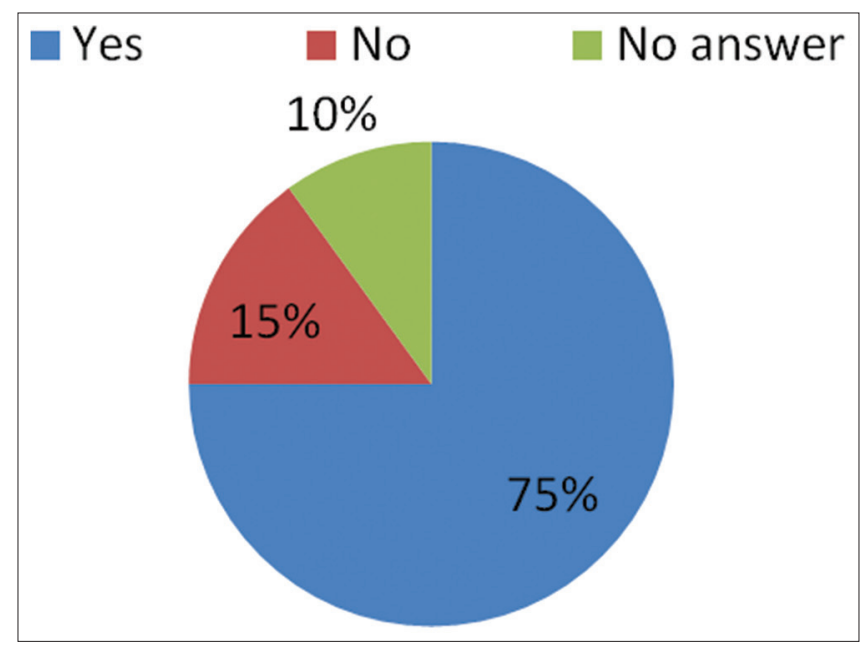

Figure 1. Pie chart of percentage of code-switching

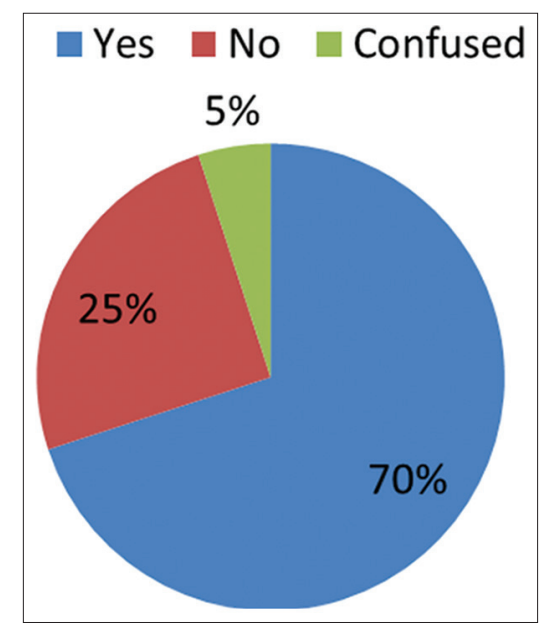

Figure 2. Pie chart of percentage of thinking regarding codeswitching negatively that actually code-switching doesn't happen frequently. Same result is found like the $1^{\text {st }}$ answer. 5\% of them were in confusion (See Figure 2).

Question no.3- How do you approach the code-switching? Data analysis: When they have asked to answer how they approach code-switching then, most of them (80\%) have answered positively and $15 \%$ have answered negatively. Others (5\%) answered that they don't know what should be the approach (See Figure 3).

Question no.4- Do you think that young are more tentative to switch the code?

Data analysis: The most important question regarding this study was asked and in this section $60 \%$ of students have said that, it is seen that to switch the code more than other generation. In comparison to previous answers this time the percentage of "No" was more in percentage (35\%). And 5\% of them don't know about it (See Figure 4).

Question no.5- When do you switch the code mostly?In formal or informal situation?

Data analysis: It is seen that the student used to switching the code in informal situation mostly (50\%). And in formal situation code-switching is used by $25 \%$ of students. Whereas, $25 \%$ of them are used to switching the code in both formal and informal situation (See Figure 5).

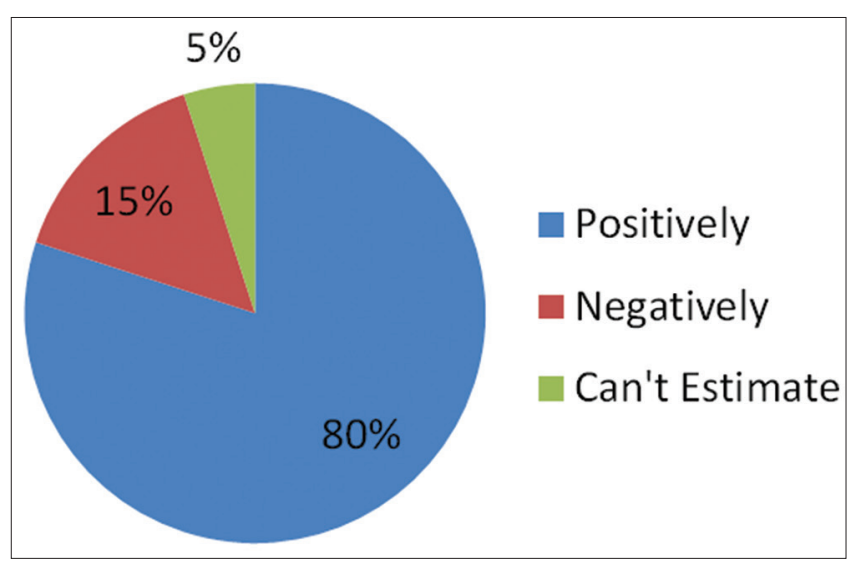

Figure 3. Pie chart of the percentage of approach of the participants

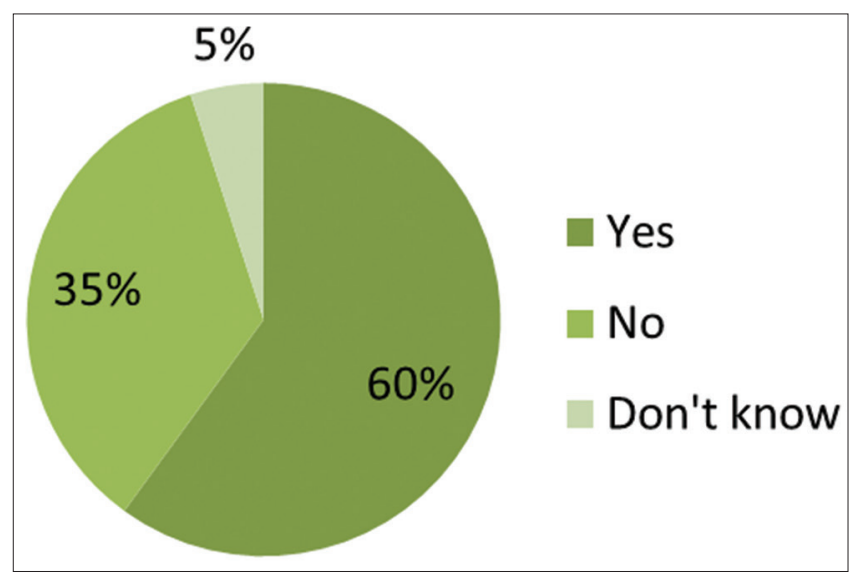

Figure 4. Pie chart of the percentage of thinking regarding young's code-switching 
Question no.6- How do you feel when someone switch the code?

Data analysis: The question was asked among 20 students that how they feel when someone switch the code. In their answer they have told mostly $(60 \%)$ that it seems to them as normal. And $20 \%$ of them feel that code-switching is odd. And other 20\% have said that they are confused with it (See Figure 6).

Question no.7- Do you think that code-switching has a negative impact on your mother tongue?

Data analysis: To answer this question, the students have felt a glimpse of nationalism. $85 \%$ of them think that code-switching have negative impacts over mother tongue. And $10 \%$ of them felt that it may have some negative impacts over mother tongue. And only 5\% of them think that code-switching have no negative impacts over mother tongue (See Figure 7).

Question no.8- Do you think that two languages together have created a new way of communication?

Data analysis: To answer this question, $70 \%$ of students said that code-switching has created a new way of communication. $20 \%$ of students have showed negative attitude and $10 \%$ of them have said that it may create a new way of communication (See Figure 8).

Question no.9- What kind of code-switching you do most? Inter-semeotic, Intra-semeotic or Tag- switching? (This question is asked after make them understand about the terms)

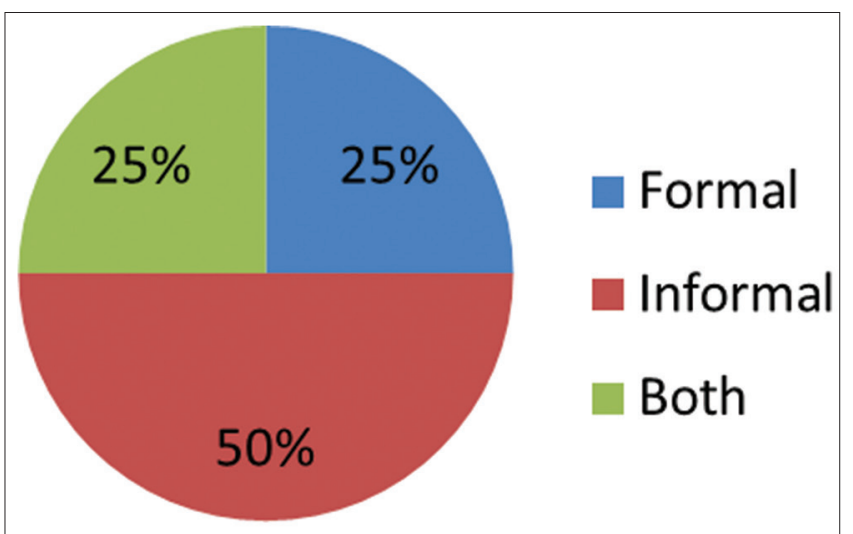

Figure 5. Pie chart of the percentage of saying about situation

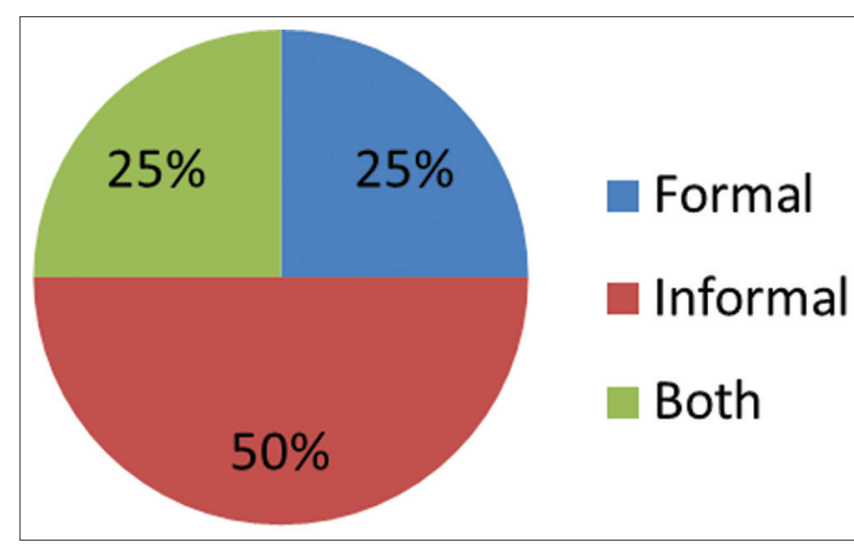

Figure 6. Pie chart of the percentage of feeling of the participants
Data analysis: The participants of the study have answered the question in a way that showed about $48 \%$ of them used to use Intra-semiotic switching; and $32 \%$ of them used to use Inter-semiotic code-switching mostly and others (20\%) usually use Tag code-switching (See Figure 9).

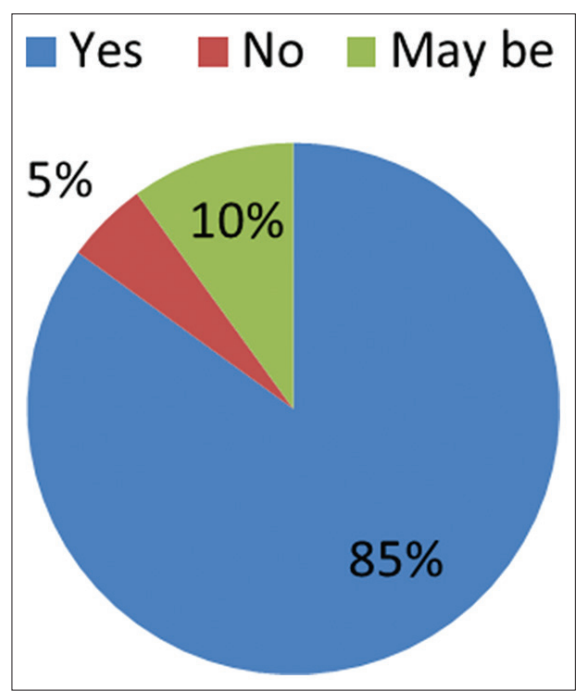

Figure 7. Pie chart of the percentage of thinking about the impact

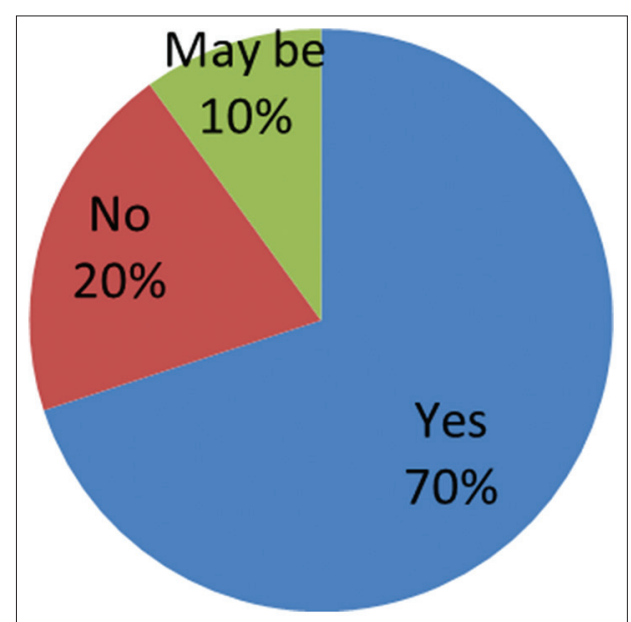

Figure 8. Pie chart of the percentage of thinking about a new way of communication

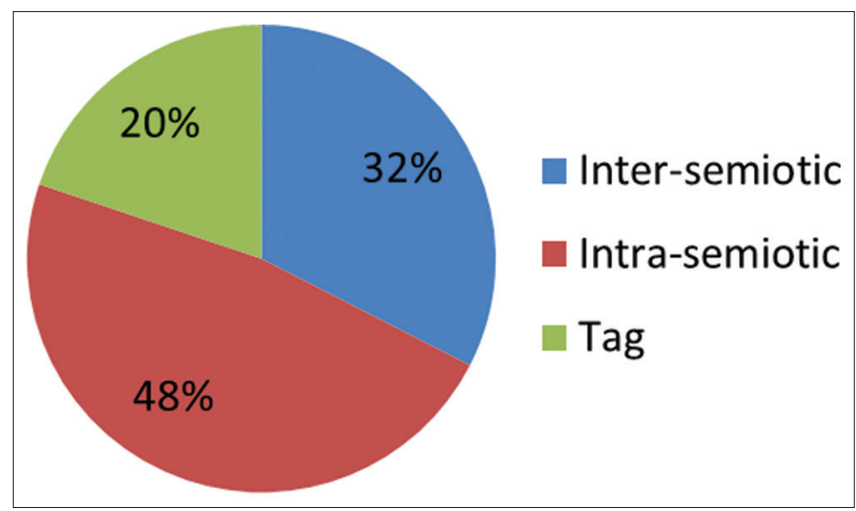

Figure 9. Pie chart of percentage of different types of codeswitching 


\section{Open-ended Question Section}

In this particular section, the students are asked several open-ended questions to surmise the perception and thinking regarding code-switching. The questions and answers are given below- (pseudonyms are used)

Question: Why do you switch the code in different circumstances?

*Abdullah: Actually I used to switch the mostly in the formal situation. When I talk to my teachers, I used to switch the code but with my friends I don't like to switch the code.

*Mehedi: I do take tuition that is why every time I remain in an English language based situation. And in my university the medium of education is English, that is why I used to switch the code. And now it becomes normal for me.

*Ratul: Sometimes English can convey my message better than Bengali language. For example, "awesome". That is why I used to switch the code.

*Farhana: In our conversation, we start the conversation in Bengali but then we shift to English. It's a habit.

*Students of English discipline, Level 4; Semester-II

Like them this question is asked among 10 students.

Everyone has their own reason to switch the code.

Question: Which language do you use most? Explain briefly

The answer of this question was mostly English and Bengali. And some were saying that they use Hindi (rarely) in their conversation. Every participant has given his own reason to use particular language. But the percentage of saying English and Bengali is large.

\section{Interview Section}

The interview section has been conducted among the teachers to know about their experiences with code-switching. How far the code-switching is good and the impact of code-switching is wanted to know from them. It could be better if the interview will be taken among more teachers but for the lack of time, the interview is conducted among five teachers from Hajee Mohammad Danesh Science \& Technology University, Dinajpur and other reputed universities in Bangladesh as well. A recorded interview is given below(the interview is translated into English)

Interviewer: Hello Sir. I am doing a survey on code-switching. Could you please help me by giving your valuable opinion?

Teacher: Of course. What do you want to know?

Interviewer: What do you think about code-switching?

Teacher: Well, as code-switching is a very common phenomenon, it should not be thought of as an alienating consequence, and now it has become a part of our daily conversation.

Interviewer: Do you switch the code in your class?

Teacher: It depends on the situation. When I feel to switch the code, I do it.

Interviewer: How your students react when you switch the code?
Teacher: I didn't find any odd behavior while I switch the code. Rather, they took it naturally.

Interviewer: Are the young generation more tentative to switch the code? What do you think sir?

Teacher: Yes, it can be because they have takes this code-switching as a trend.

Interviewer: In the education sector how code-switching could work?

Teacher: I think that in the education sector it will help the students to understand a thing. But sometimes it becomes a hassle when any teacher doesn't like to switch the code.

Interviewer: Do you think that psychology is related to code-switching?

Teacher: Yes, It is. When you feel that you need to switch the code, then it will automatically come into your mind.

Interviewer: Thank you, Sir, for giving your valuable time.

Teacher: Welcome.

\section{Patterns of the Interview}

The entire interview section has conducted among 5 teachers but here only one sample is given. The entire journey of interview is shown by a bar diagram-(teacher's attitude is rated in the scale of $10 \&$ " $\mathrm{T}$ " means teacher here).

Answer analysis: The interview section was held among 5 teachers and the question type was open-ended question. The teachers are asked several questions and from their answers a general hypothesis has been drawn. Their experience and attitude towards code-switching is rated under the number 10. Among 5 teachers 3 of them have shown very positive attitude. It is rated $10 / 10$ and among them a teacher has shown mostly positive attitude. They are rated 9/10 and another teacher who have showed some positive attitude and some negative attitude. That is why he is rated 5/10 (See Figure 10).

\section{Psychological Aspect regarding Code-switching}

The paper aims at finding the psychological element by which code-switching is accelerated. Surprisingly, most of the participants switch the code top English or Arabic which is a prestigious variety. It is because of social acceptance, showing smartness, better understanding etc. As code-switching is related to language change then, there is a close relation between code-switching and the psychology of the emerging youths. When someone switches the code very frequently, then it means that he/she is very adept in foreign language

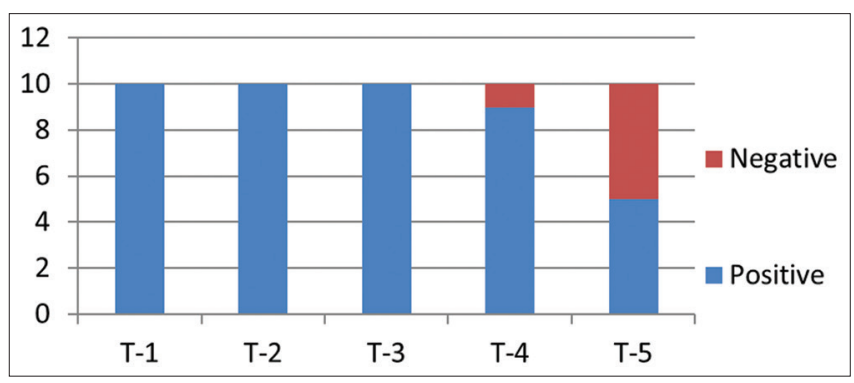

Figure 10. Bar diagram for code-switching attitude of the teachers 
(like-English). Then it becomes a matter of prestige and show-off. In Bangladeshi context, if anyone deliberately speaks English then it becomes a prestigious thing for him/ her. And people used to pay respect over them and that is why a code-switching tendency is created among the youngsters that they have to look themselves as in a superior position. Another psychological aspect is related to code-switching which is to get attention of anyone. In Bangladeshi context, switching-code is seen as an element of smartness. To attract the opposite sex through message or through conversation, code-switching really works that is why an ideology is created in the mind of the speaker that whenever he/she will get a chance, he/she will switch the code.

The participants are asked several questions regarding the psychological aspects. The questions are given below:

I. When you like to switch the code mostly? Is it before your sex or opposite sex?

II. Do you switch the code when you meet a new person?

III. Do you think code-switching is a tool of showing smartness?

IV. Is it possible to impress the opposite sex by switching code?

V. Do you think that code-switching is a trend?

These 5 questions are asked to the participants to know about their psychology regarding code-switching. All the questions are linked with the mind of the youngsters. The answers of the questions are analyzed and discussed below-

I. A variety of answers is got from the participants. Like, some said that, "yes, sometimes I used to do it but not all the time." Another person says that he never did it and someone said that, "everyone does it but nobody reveals the thing." Hence, it is said that maximum young prefer to switch the code before opposite sex more than same sex.

II. In the literature review section, it is described by Holmes that an arrival a new person forces the speaker to switch the code. And the answers from the students were almost same. Among them most of the students have distinguished the fact that a person switches the code when he/she faces a new person.

III. About $99 \%$ answers of this question were positive. They think that switching the code is a tool of showing smartness. Additionally they have said that most of the young students think like that way.

IV. The answers of this question have some variations. Some of them think that it is possible. Most of them said that it is possible. No one has said that it is impossible rather they said that it can be possible.

V. Young generation is more aggressive to follow the trend that is why the answer was expectedly positive among the 20 students.

Therefore, from the above discussion, it can be concluded that code-switching is more or less a typical phenomenon. The young generation is more likely to switch the code for establishing themselves in a superior position. And the psychology of the speaker accelerates the code-switching in different circumstances. To show off and to be smart the young frequently switch the code who have enough knowl- edge regarding more than one language.

\section{Limitation of the Study}

The paper only consists of the case study of 20 students and 5 teachers as the researcher has conducted the study for the partial fulfillment of his graduation degree. Thus, the time was very short to conduct the study among the students of all the universities in Bangladesh. Then again, the students and teachers are from more or less same socio-economic background. Therefore, there is a high possibility of skewed data. However, the study is conducted from a neutral position, keeping all the associated factors in the mind, if the researcher had enough time, he would have been more accurate and specific.

\section{FINDINGS, RESULTS, AND RECOMMENDATION}

\section{Findings}

From the questionnaire section, by examining the answers to the given questions several points have emerged. The questionnaire section shows that most of the students have taken code-switching positively. And, there are a few students who have been confused about it. The using rate of code-switching among the students is high. They usually switch the code in both formal and informal situations as well.

The socio-linguistic advantages of code-switching comprise communicating solidarity with or association to a distinct social group and so code-switching can be inspected as a means of rendering a linguistic advantage rather than a barrier to communication. Moreover, code-switching permits a speaker to conduct more subtle attitudes and sensations by adopting from a bigger pool of words that are accessible to a bilingual person, much like how one might use a font, bolding, or underlining in a text document to highlight purposes. Employing the second language, then, permits speakers to increase the impact of their speech and use it more efficiently. The participants are more like to use intra-semiotic code-switching which occurs within a sentence or clause. That means the youths are aggressive to find out the easy way of code-switching but they are not actually aware of that, rather it becomes a spontaneous occurrence.

As the attitude towards the code-switching is seen positively, they have told that they are usually used to switching the code in their classroom, hangout, and in other functions. How they used to switching the code that is shown on the "Patterns of code-switching" section.

By analyzing the interview questions and answers, the attitude of the teachers towards code-switching can be assumed. By interviewing the teachers, it is seen that most of the teachers have shown a positive attitude towards code-switching. Most of the teachers used to switch the code in their classroom or other formal and informal circumstances. They have taken code-switching as a natural phenomenon. And, only one teacher has only shown both positive and negative attitudes towards code-switching. But, at the overall discussion and from the pie chart and bar diagram, it can be seen that a positive and natural outlook towards code-switching. 


\section{Results}

The result of this study is determined by investigating on the primary and secondary sources. Mostly, the paper depends on the questionnaire section and interview section. This field work has helped to find out the real nature of code-switching propensity among the young. Along with the questionnaire section, some students have made some comments. It will be very easy to go into a conclusion if it is analyzed the student's comment. Hence, they are listed below:

Comment of some Respondents in the Questionnaire

a) Bengali is our mother tongue. In our conversation English should be avoided.

b) I feel easy and comfortable in conversation by switching the code.

c) As English as an international language then, we should adopt more and more English, and have to use it in our conversation.

d) Mixing Bengali and English is not a matter of feeling satisfied-it is a matter of easy communication.

e) I think that to deal with this competitive world it is very normal to switch the code.

f) Between Arabic language and Bengali language code -switching is normal as Arabic should be studied in purpose of religion but code-switching between Bengali and English is not normal.

g) I think that the student of public university switch the code mostly.

h) Code-switching is good but sometimes it becomes deformed by some people. That's really irritating.

i) It becomes spontaneous for me now to switch the code.

j) It is a psychological fact. It depends on situation.

From these comments and findings, it can be supposed that the people of Bangladesh habitually switch the code very frequently. However, the most educated people contemplate it as very natural and positive phenomenon. On the other hand, it is usual that the perspective can be different from one another. Furthermore, some are saying that with the advancement of the world, it is a very typical phenomenon. In one perspective code-switching is relevant to Bengali and English but between Hindi and Bengali code-switching is not relevant. But if code-switching can be taken place between Bengali and English then it is inevitably relevant that code-switching also can be taken place between Hindi and Bengali or between Bengali and Arabic. Hence, all things depend on particular consequence and setting. It is noticed that, while switching code the youths switch the code to English because English can be considered as a prestigious variety that is called Acrolect. And then, if it is seen in the Arabic Code-switching then it can be noticed that Arabic also a prestigious variety for Muslims also. Therefore, it can be reached at the resolution that it is a psychological aspect to switch to the acrolect while switching the code.

\section{Recommendation}

From the overall data analysis, findings, and results it comes to light that in Bangladesh not everyone is willing to switch the code and almost all the people switch to acrolect. It is observed that the students are doing some surprising comments that tell that there is a lot of confusion with code-switching among the Bangladeshi emerging youths. Some basic things should keep in mind while a speaker is switching the code. As this is a psychological fact then it depends on the mind of the speaker. Sometimes, it should be avoided to switch the code. The speaker, at first, should know about the context and setting and then he/she should decide whether he/she will switch the code or not. For example, if the speaker is in a government office or in a viva-voce, then code-switching must be prohibited. As code-switching is related to bilingualism, the speakers should have a clear knowledge about languages they are using. It should not switch the code if the speaker has less proficiency in any language between the two. Besides, the deformed code-switching should be avoided. Though code-switching is a common phenomenon; the speaker should not always switch the code.

\section{How the Youths can safely adapt to Code-switching}

As the entire study is related to emerging youths and code-switching then it is mandatory to have a possible solution about how the youths can safely adapt to code-switching. From the data analysis, it is seen that the young have already a positive attitude towards code-switching. But to avoid debate, some possible solutions can be provided from the hypothesis of the data analysis result.

Firstly, young speakers should have a lucid knowledge in more than two languages. Otherwise, they can't be able to adapt to code-switching very easily.

Secondly, they must take the code-switching naturally. When they can be able to take it as normal consequences then it will be very easy to adapt to code-switching.

Thirdly, the young should have a lucid idea about the context. Whether in this context code-switching is safe or not that should be understood.

Fourthly, they should not switch the code all the time of their conversation. Code-switching should have a limit. They should not cross the limit.

Fifthly, it is better to avoid code-switching in the formal situation but if the context is favorable then they can switch the code.

Sixthly, if a young speaker has a very poor knowledge regarding the foreign language then he/she should not switch the code.

Seventhly, they have to switch the code in such a way that it doesn't make any negative impact over their mother tongue.

Eighthly, the young speaker must have to understand the mind of their speaking partner. Then, it will be very easy for them to switch the code.

Last but not the least; they should have to make a favorable image by switching the code.

Therefore, this paper has found some possible solution of adapting to code-switching safely and it hopes that will make the code-switching as an easy tool of expressing someone's feelings, thinking, and opinions. 


\section{CONCLUSION}

Throughout the above discussion, it can be found that code-switching has become a very ecumenical phenomenon not only among the youths of Bangladesh but also among all the age-grouped of people. The youths are willing to switch the code very frequently and the study shows a positive attitude towards code-switching and the youths switch the code to acrolect most of the time. Different types of psychological discourse are responsible for the code-switching as it is seen that code-switching is related to language and mind. From the questionnaire and interview along with some open-ended questions, it is made clear that a variety of thinking roams around the mind of the participants but the overall results show an unleashing effect of code-switching among the emerging youths of Bangladesh. Besides, it is seen that balanced bilingual speakers are very much adept in code-switching and they frequently switch the code. Moreover, the study has analyzed the repercussion of code-switch among the participant students and teachers. The impact of code-switching is that it has made many things easy to express when there has no parallel word found in a language then, code-switching becomes a mandatory way to cope up with the circumstances. From the psychological point of view, the youths switch the code mostly for maintaining the spontaneity or to attract the opposite sex and the data analysis says that among many youths, some have considered code-switching as a habit. It is also seen that the youths used to use Intra-semiotic code-switching mostly. Moreover, mind has lucid relation with language that is why it is seen that when a person meets with someone as new, a tendency creates in his/her mind to switch the code. Though Bangladesh is not a bilingual country, the propensity of switching the code has opened a new way of communication system and hopefully the tendency leads the youths to make them acquainted with a new dimension of bilingualism as well as multilingualism. It is recommended in the recommendation section in this paper, some possible way by which the youths can safely adapt with code-switching environment. This study also found out that maximum young are tentative to switching the code from Bengali to English and vice-versa but a propensity to switching the code from Bengali to Hindi or Arabic is also remarkable so far.As the study consists of qualitative and quantitative approach, it has maintained a reliable data collection procedures and it has tried to conduct the study from a neutral point of view. Since the aim and goals of the study started with why code-switching is necessary and how acrolect operates as a catalyst of code-switching, the study accomplished all the points through data collection and discussion. And it is found that code-switching is necessary for adaptation, social acceptance, comfortable zone etc. and acrolect which plays very massive role because of the speakers tend to switch the code to acrolect. Hence, the overall results and findings of the study will help the future researches to conduct the study more broadly and the data analysis and other elementary things will expand the nature and scope of the code-switching in sociolinguistics ground of study.

\section{ACKNOWLEDGMENTS}

Firstly, I would like to thank Almighty Allah just for being with me all the way and giving me the strength and patience to complete my thesis. I would like to thank my supervisor sir who has supported me in all the way to complete my thesis. I am also thankful for his concern, careful guidance, and proper advice, feedback that he has given me showing his patience. Then I would like to thank to all the teachers and my friends who have directly or indirectly supported and helped me in various ways to do this thesis.

I am thankful to my parents who have encouraged me and helped me with money when I needed.

\section{REFERENCES}

Ahmed, S., \& Tinny, F. Z. (2011). The RJ style: Brought to you by Bangladeshi FM Radio. Stamford Journal of English, 6, 1-24.

Alam, S. (2006). Code Mixing in Bangladesh: A case study of non-government white-collar service holders and professionals. Asian Affairs, 28.

Banu, R., \& Sussex, R. (2001). Code-switching in Bangladesh. English Today, 17 (2), 52-53.

Chomsky, N. (2006). Language and Mind. Cambridge, New York: Cambridge University Press.

Chowdhury, N. (2013). Classroom Code Switching of English Language Teachers at Tertiary Level: A Bangladeshi Perspective. Stamford Journal of English, 7, 41.

Chui, H. L., Liu, Y., \& Mak, B. C. (2014). Code-switching for newcomers and veterans: a mutually-constructed discourse strategy for workplace sociolization and identification. International Journal of Applied Linguistics , 4.

Grosjean, F. (1982). LIFE WITH TWO LANGUAGE-An Introduction to Bilingualism. Cambridge: Harvard University Press.

Gumperz, J. J. (1982). Discourse Strategies. Cambridge: Cambridge University Press.

Holmes, J. (2001). An Introduction to Sociolinguistics. New York: Routledge Taylor \& Francis Group.

Hudson, R. A. (2007). Sociolinguistics. New York: Cambridge University Press.

Inuwa, YN; Christopher, AA; Bakrin, H BT;. (2014). Factors motivating code switching within the social contact of hausa bilingual. IOSR Journal of Humanities ans social science, 19, 43-49.

Kaziol, J. M. (2000). Code switching between Spanish and English in contemporary american society. MS thesis, St. Mary's college of Maryland.

Nilep, C. (2006). "Code Switching “ in Sociolicultural Linguistics. Colorado Research in Linguistics, 19, 22.

Sultana, N. (2010). English code switching in Bangladeshi commercial cinema. International Journal of Social Science and Humanities Research, 1, 40-43.

Wardhaugh, R. (2010). An Introduction to Sociolinguistics. West Sussex: Wiley-Blackwell.

Wei, L. (2000). The Bilinguialism Reader. London: Routledge Taylor \& Francis Group. 